\title{
Study on Reactive Power Optimization of Active Distribution Network Based on Bifurcation Theory
}

\author{
Xianbin Dai \\ Shenyang Institute of Engineering \\ Shenyang, China \\ dxbcy@126.com
}

\begin{abstract}
As the basic method used to analyze the stability of nonlinear dynamic system, it is able to more deeply discuss the stability problems of power system in the vicinity of a critical point in comparison with traditional analysis method. Active power distribution network is a complicated nonlinear dynamic system. The change of voltage stability is the process to convert from stable status to bifurcation in essence. Taking 10-node active distribution system as an example, the author studies the influence of reactive power optimization on voltage stability based on bifurcation theory. The author explains the reactive optimization algorithm based on numerical simulation, namely, it is the decision - making algorithm in order to realize multiple purposes of having minimum power network loss, largest reactive power margin with stable operation of power distribution network and minimum voltage floating in system fluctuation, which is proved to be effective in elevating power distribution system stability, reducing power loss and improving voltage quality.
\end{abstract}

Keywords-reactive power; optimization; active distribution network; bifurcation theory

\section{INTRODUCTION}

In front of the huge challenge brought by global energy saving, emission reduction and sustainable development of power, the development of distributed type of power generation based on renewable energy has become the necessary choice. Nevertheless, the introduction of distributed energy in large amount will create wide-ranged influence on power distribution network as follows: it alters the voltage level of distribution network, elevates the short-circuit capacity of distribution network, increases the complex of relay protection strategy, influences network power supply reliability and accelerates the deterioration of power quality, etc. [1-4] Besides, the characteristics of distributed power of being random, intermittent, floating and hard to control restrains power grid from digesting and absorbing the distributed type of power generation.

Synergic control has become the necessary function for modern power distribution network operation and control system. It requests power distribution network to convert from the traditional reactive power grid to the active power distribution network, which is able to conduct active adjustment and participate in power grid operation and control based on practical operation status of power grid. Active distribution network is the distribution network that is able to compose and control various distributed energy abilities (DG, controllable load, energy storage and demand-side management, DSM, etc) and it intends to enlarge the distribution network's ability to accept the renewable energy, to elevate the use rate of distribution network assets, to slow down the upgrading and investment on distribution network and to improve users' power use quality and power supply reliability.

In active power distribution network, the introduction of distributed energy in large amount will bring severe challenge for reactive voltage. Urged by the limitation of energy shortage and pressure of environmental protection, the operation of active power distribution network is approaching its limit status and problems of voltage stability might happen at any time. In recent years, voltage stability accidents taking voltage collapse as the characteristics keep happening in distribution networks in the world and the accidents indicate that the system's reactive distribution and load's nonlinear dynamic features have obvious influence on voltage stability.

A large amount of files and simulation analyses indicate that the reasonable distribution of reactive power rate is the most effective way to guarantee voltage stability, which has equally important role as synch operation stability and frequency stability but its accidents contain characteristics of "sudden outbreak" and "hidden performance" in comparison with the synch operation stability and frequency stability. Besides, reactive voltage control in active distribution network needs also to consider the coordinated cooperation by reactive resources in distributed power supply, which needs to realize two purposes of energy saving and guaranteeing voltage quality at the same time. Bifurcation theory is an important branch in nonlinear science and it mainly studies the process of changes of solution structure and stability caused with the change of parameters in the system. Active distribution network is a complicated nonlinear dynamic system and the change of voltage stability is the process of converting from stable status to bifurcation. Therefore, bifurcation theory is the effective tool to analyze the structural stability mechanism analysis in active distribution network. [5-8] Establishing the active distribution network element model and using bifurcation theory to analyze the influence of load dynamic characteristics on voltage stability contain important meaning 
for deeply recognizing the dynamic essence of power system and understanding the influence of load dynamic characteristics on voltage stability.

Active distribution network stability based on bifurcation theory.

As to a nonlinear dynamic system, its losing stability and collapsing belong to one nonlinear dynamic process. In essence, when parameters keep changing and pass one critical value, it is necessary for system to create sudden qualitative behavior and it is called bifurcation and the corresponding balance point is called bifurcation point. In the view of match, the dynamic behavior in distribution network can be described in differential equation as follows:

$$
\begin{aligned}
& \dot{x}=f(x, y \cdot \mu) \\
& 0=g(x, y, \mu)
\end{aligned}
$$

In the formula, $\mathrm{f}$ is the differential equation used to describe dynamic behaviors such as motor and load in the distribution network; $g$ is algebraic equation used to describe reactive and active rate on each node; $\mathrm{x}$ is system state variable; $\mathrm{y}$ is system algebraic status variable; $\mu$ is system control parameter.

In order to study dynamic stability of distribution network, it conducts differential change in formula (1) at the balance point $\left(\mathrm{x}_{0}, \mathrm{y}_{0}, \boldsymbol{\mu}_{0}\right)$ as follows and we can obtain:

$$
\left\{\begin{array}{l}
\frac{d x}{d t}=D_{x} f\left(x_{0}, y_{0}\right) d x+D_{y} f\left(x_{0}, y_{0}\right) d y \\
0=D_{x} f\left(x_{0}, y_{0}\right) d x+D_{y} f\left(x_{0}, y_{0}\right) d y \\
A=D_{x} f\left(x_{0}, y_{0}\right) \\
B=D_{y} f\left(x_{0}, y_{0}\right) \\
C=D_{x} g\left(x_{0}, y_{0}\right) \\
D=D_{y} g\left(x_{0}, y_{0}\right) \\
J=\left[\begin{array}{ll}
A & B \\
C & D
\end{array}\right]
\end{array}\right.
$$

We can simplify formula (2) and obtain:

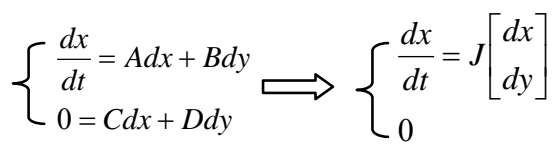

Then differential equation of system with dynamic characteristics is as follows:

$$
\frac{d x}{d t}=\left(A-B D^{-1} C\right) x=J x
$$

The dynamic characteristics of system can be totally determined by characteristic value of Jacobian matrix. In bifurcation theory, we can identify different bifurcation points (tracing equilibrium solution manifold of system and search for bifurcation point) based on the changes of characteristic value of Jacobian matrix with the change of control parameters.

Bifurcation phenomena in distribution network are complicated and diversified and the three common categories are as follows: saddle node bifurcation, Hopf bifurcation and singularity-induced bifurcation. The saddle node bifurcation is the most common partial static bifurcation in distribution network and it is the bifurcation with the disappearance of balance point in vector field and its bifurcation phenomena contain linear matrix with determinant at zero in the vector field. Hopf bifurcation is related to unstable oscillation of system. In one Hopf bifurcation, one balance point interacts with one limit circle, which makes the stability of balance point disappear. The singularity-induced bifurcation is the special kind of bifurcation occurred when constraint manifold and obstacle surface interact. Currently, there is no uniform theory related to strict mathematical definition of the singularityinduced bifurcation both at home and abroad.

\section{REACTIVE OPTIMIZATION OF ACTIVE DISTRIBUTION NETWORK}

Reactive optimization of active distribution network is to reduce the system active power loss through the control of reactive power emitted by power supply and guarantee the node voltage remain in the regulated range. The study intends to realize multiple purposes of having minimum power network loss, largest reactive power margin with stable operation of power distribution network and minimum voltage floating in system fluctuation. The multi-purpose optimization module contains three parts such as object function, flow constraint equation and variable constraint condition.

Object Function: minimum power network loss, largest reactive power margin with stable operation of power distribution network and minimum voltage floating in system fluctuation

$$
\left\{\begin{array}{l}
B_{1}=\sum_{i=1 j=1}^{n} G_{i j}\left(U_{i}^{2}+U_{j}^{2}-2 U_{i} U_{j} \cos \theta_{i j}\right) \\
B_{2}=\sum_{i \in q}\left|Q_{q i}\right| \\
B_{3}=\sum_{i=1}^{n} \frac{U_{i}^{s p e c}-U_{i}}{U_{i \max }-U_{i \min }}
\end{array}\right.
$$

In the formula, $B_{1}$ is network loss; $B_{2}$ is reactive power margin; $B_{3}$ is voltage floating; $U_{i}, U_{j}$ refer to voltage amplitude at node $i$ and $j, U_{i j}$ is conduction between node $i$ and $j, \theta_{i j}$ is voltage phase angle difference between node $\mathrm{i}$ and $\mathrm{j} ;{ }_{j}^{U^{\text {spec }}}$ is node voltage $\mathrm{BLV} ; \mathrm{Q}_{\mathrm{qi}}$ is reactive compensated capacity of node $\mathrm{i}$

Flow Constraint Equation: in order to guarantee the system safe operation and power quality, the constraint conditions of object function are divided into flow constraint equation constraint and operation limit constraint. The constraint conditions for the balance between active power and reactive power in each node is as follows:

$$
\left\{\begin{array}{l}
P_{G i}=U_{i} \sum_{j=1}^{n} U_{j}\left(G_{i j} \cos \theta_{i j}-B_{i j} \sin \theta_{i j}\right) \\
Q_{G i}=Q_{i j}+U_{i} \sum_{J=1}^{n} U_{j}\left(G_{i j} \sin \theta_{i j}-B_{i j} \cos \theta_{i j}\right)
\end{array}\right.
$$

In the formula, $\mathrm{P}_{\mathrm{Gi}}$ is power generator's active output in node $\mathrm{i} ; \mathrm{B}_{\mathrm{ij}}$ is electrical susceptance between node $\mathrm{i}$ and $\mathrm{j} ; \mathrm{G}_{\mathrm{ij}}$ is 
conduction between node $\mathrm{i}$ and $\mathrm{j} ; \mathrm{Q}_{\mathrm{Gi}}$ is motor reactive output in node $\mathrm{i}$;

Variable Constraint Condition: variable in distribution network's reactive optimization can be divided into control variable and status variable. Reactive power emitted by distributed power supply belongs to control variable and each load node voltage belongs to status variable. The introduction of distributed power generation is to transmit active power to power grid. When reactive power is too large, the capacity of grid-connected inverter will become very large. In the angle of economy, the capacity of reactive power shall be limited.

Constraint condition for control variable is as follows:

$$
\left\{\begin{array}{l}
Q_{C j \min } \leq Q_{C j} \leq Q_{C j \max } \\
U_{G i \min } \leq U_{G i} \leq U_{G i \max }
\end{array}\right.
$$

In the formula, $\mathrm{U}_{\mathrm{G}}$ is terminal voltage of power generator; $\mathrm{Q}_{\mathrm{c}}$ is amount of compensation capacitors

Constraint condition for status variable is as follows:

$$
\left\{\begin{array}{l}
Q_{G i \min } \leq Q_{G i} \leq Q_{G i \max } \\
U_{D j \min } \leq U_{D j} \leq U_{D j \max }
\end{array}\right.
$$

In the formula, $Q_{c}$ is power generator's reactive output; $U_{D}$ is voltage between load node $\mathrm{i}$ and $\mathrm{j}$.

\section{SimULATION VERIFICATION OF NUMERICAL EXAMPLE}

In order to verify the optimization effect of multi-purpose decision-making algorithm based on bifurcation theory (Bifurcation Theory Optimization,BTO), taking distribution network composed of full outlet $10 \mathrm{kV}$ in one power substation as an example, the author conducts Matlab7.0 programming and optimization calculation. In this distribution network, it contains 10 nodes, among which node 3,7 and 9 are reactive compensation nodes. Compare the node calculation result with PSO (Partiele Swarm Optimization, PSO; momentum factor from 0.4 to 0.9 and acceleration coefficient is 2 ).

The values of original parameters $\delta m \omega, \theta, \mathrm{u}$ in multi-purpose decision-making algorithm based on bifurcation theory (BTO) are respectively at $0.3,1.5,0,2$ and 0.95 and the simulation optimization result is as illustrated in Table 1,2 and 3 as follows.

TABLE 1. Power Grid Loss before Distribution Network Compensation $(\mathrm{kW})$

\begin{tabular}{cccc}
\hline Indicator & $\begin{array}{c}\text { Before } \\
\text { Compensation }\end{array}$ & PSO & BTO \\
\hline $\begin{array}{c}\text { Power Grid } \\
\text { Loss }\end{array}$ & 397.23 & 164.61 & 143.21 \\
\hline
\end{tabular}

TABLE 2. Compensated Capacity at Compensation Node in Distribution Network (kvar)

\begin{tabular}{ccc}
\hline $\begin{array}{c}\text { Compensation } \\
\text { Node }\end{array}$ & PSO & BTO \\
\hline 3 & 412.53 & 320.71 \\
\hline 7 & 385.87 & 367.32 \\
\hline 9 & 497.65 & 416.36 \\
\hline
\end{tabular}

Based on Table 1 and 2, we can see that multi-purpose decision-making algorithm based on bifurcation theory (BTO) has small compensation capacity with good loss reduction effect, which not only reduces investment but also reduces operation expense. Conducting the iteration for several times by using BTO will gradually realize the purpose of stability. The adoption of PSO contains small decrease amount in iteration in each time and it can only realize stability after it conducts iteration over 30 times, which indicates that multipurpose decision-making algorithm based on bifurcation theory (BTO) has better convergence rate.

TABLE 3. Voltages at Each Node in Distribution Network Before/After Compensation (p.u)

\begin{tabular}{cccc}
\hline Node & $\begin{array}{c}\text { Before } \\
\text { Compensation }\end{array}$ & PSO & BTO \\
\hline 1 & 0.961 & 0.997 & 0.998 \\
\hline 2 & 0.952 & 0.989 & 0.993 \\
\hline 3 & 0.948 & 0.971 & 0.989 \\
\hline 4 & 0.943 & 0.962 & 0.971 \\
\hline 5 & 0.939 & 0.953 & 0.968 \\
\hline 6 & 0.921 & 0.940 & 0.957 \\
\hline 7 & 0.927 & 0.945 & 0.958 \\
\hline 8 & 0.929 & 0.941 & 0.949 \\
\hline 9 & 0.923 & 0.938 & 0.941 \\
\hline 10 & 0.918 & 0.929 & 0.938 \\
\hline 11 & 0.914 & 0.920 & 0.926 \\
\hline 12 & 0.910 & 0.915 & 0.920 \\
\hline 13 & 0.906 & 0.908 & 0.912 \\
\hline
\end{tabular}

Based on Table 1 and 2, we can see that multi-purpose decision-making algorithm based on bifurcation theory (BTO) has small compensation capacity with good loss reduction effect, which not only reduces investment but also reduces operation expense. Conducting the iteration for several times by using BTO will gradually realize the purpose of stability. The adoption of PSO contains small decrease amount in iteration in each time and it can only realize stability after it conducts iteration over 30 times, which indicates that multipurpose decision-making algorithm based on bifurcation theory (BTO) has better convergence rate.

Based on Table 1 and 3, we can see that network loss after compensation is reduced from $397.23 \mathrm{~kW}$ to $143.21 \mathrm{~kW}$ and it creates obvious loss reduction effect. Voltage at each node after compensation is elevated and the voltage at lowest node rises from 0.912 to 0.938 , by which it largely improves the voltage quality indicator in distribution network.

\section{CONCLUSION}

The paper puts forward the active distribution network stability and reactive optimization method with multi-purpose decision-making algorithm based on bifurcation theory (BTO), which optimizes system network loss, voltage differentiation and reactive power compensation capacity's margin in order to obtain the satisfactory solution. Compare the node calculation result with the PSO algorithm and we obtain that the method contains characteristics of having small system active network loss, small voltage differentiation, small reactive compensation capacity and high margin of static voltage stability, etc. 


\section{ACKNOWLEDGMENT}

The paper is sponsored by "SIE Technology Fund - General Project (LGYB-1414)".

\section{REFERENCES}

[1] Cunlu Dang, Qingfeng Wu, Influence and Improvement of Distribution Network's Power Quality by Distributed Power Supply, Electric and Electronic Technology. China, vol. 47. 2013, pp. 58-60.

[2] You Yi, Liu Dong, Wenpeng Yu, etc, Active Distribution Network Technology and Development, Electric System Automation. China, vol. 36.2012, pp.10-16

[3] Li Shan, Jitao Kang, Junfei Cui, etc, Study on Influence of Dynamic Load Characteristics on Voltage Stability Based on Bifurcation Theory, . China, Electric Technology, vol. 10. 2012 , pp.16-20.
[4] Yugang Jing, Yingqiu Li, New Methods of Calculating Electric System's Voltage Bifurcation Point, Shandong Electric Power Technology. China, vol.4. 2010 , pp. 1-4.

[5] Yi You, Chengshan Wang, Peng Li, Development Challenges of Distributed Generation, the Micro-grid

[6] and Smart Distribution System, Automation of Electric Power Systems, Vol.34 .2010, p.10-14.

[7] Shengqing Li, Lilin Zeng, Xiaodong Luo, Electric Power System's Reactive Optimization based on Multiple-objective Decision-Making Evolutionary Algorithm, Low Voltage Apparatus, Vol. 4 .2013, p.45-49.

[8] Jiguang Liu, Haiyang Wang, Lijun Zhong, Hopf Bifurcation Control Simulation of Voltage Stability in Wind Power System, Jilin University Journal (Science Edition), Vol.1 .2013, p.111-115 